\title{
Dynamic Analysis of Fractional-Order Fuzzy BAM Neural Networks with Delays in the Leakage Terms
}

\author{
Pan Wang, Jianwei Shen \\ Applied Mathematics, Xuchang University, Xuchang, China \\ Email:wp521009@126.com,xcjwshen@gmail.com
}

How to cite this paper: Wang, P. and Shen, J.W. (2017) Dynamic Analysis of Fractional-Order Fuzzy BAM Neural Networks with Delays in the Leakage Terms. Applied Mathematics, 8, 1808-1819. https://doi.org/10.4236/am.2017.812129

Received: November 1, 2017

Accepted: December 26, 2017

Published: December 29, 2017

Copyright ( $) 2017$ by authors and Scientific Research Publishing Inc. This work is licensed under the Creative Commons Attribution International License (CC BY 4.0).

http://creativecommons.org/licenses/by/4.0/

\begin{abstract}
In this paper, based on the theory of fractional-order calculus, we obtain some sufficient conditions for the uniform stability of fractional-order fuzzy BAM neural networks with delays in the leakage terms. Moreover, the existence, uniqueness and stability of its equilibrium point are also proved. A numerical example is presented to demonstrate the validity and feasibility of the proposed results.
\end{abstract}

\section{Keywords}

Fractional-Order, Fuzzy BAM Neural Networks, Uniform Stability, Delay, Leakage Terms

\section{Introduction}

Fractional-order calculus is an area of mathematics that deals with extensions of derivatives and integrals to noninteger orders and represents a powerful tool in applied mathematics to study a myriad of problems from different fields [1] [2] [3] [4]. Analogously, starting with a linear difference equation, we are led to a definition of fractional difference of an arbitrary order [5]. Nowadays, studying on fractional-order calculus has become an active research field. In recent years, fractional operator is introduced into artificial neural networks, and the fractional-order formulation of artificial neural network models is also proposed in research results about biological neurons.

The analysis of fractional-order artificial neural networks has received some attention, and some important and interesting results have been obtained [6]-[11]. For instances, the stability and multi-stability (coexistence of several 
different stable states), bifurcations and chaos of fractional-order neural networks of Hopfield type were investigated in [6]. Finite-time stability in neural networks with delay has been discussed in [7]. Zhang and $\mathrm{Yu}$ [8] proposed fractional-order Hopfield neural networks with discontinuous activation functions and investigated its stability through the Lyapunov functionals. In [9], authors considered the following fractional-order autonomous neural network:

$$
\begin{gathered}
D^{\alpha} x_{i}(t)=-C_{i} x_{i}(t)+\sum_{j=1}^{n} a_{i j} f_{j}\left(x_{j}(t)\right)+\sum_{j=1}^{n} b_{i j} g_{j}\left(x_{j}(t-\tau)\right)+I_{i}, \\
\quad i \in N=1,2, \cdots, n, t \in[0, T], T<+\infty
\end{gathered}
$$

where $D^{\alpha}$ is the fractional derivative and $0<\alpha<1 ; x_{i}(t)$ corresponds to the state of the $i$ th unit at time $t, f_{j}(\cdot)$ and $g_{j}(\cdot)$ denote the activation function of the th neuron. A sufficient criterion ensuring the uniform stability of the system and the existence, uniqueness, and uniform stability of the equilibrium point is presented.

Recently, a typical time delay called leakage delay which is the time delay in the leakage term of the systems and a considerable factor affecting dynamics for the worse in the systems, has a great impact on the dynamical behavior of neural networks. Since leakage delays can have a destabilizing influence on the dynamical behaviors of neural networks, it is necessary to investigate leakage delay effects on the stability of neural networks (see [12] [13] [14] [15] [16]). Fuzzy theory is considered as a more suitable method for the sake of taking vagueness into consideration; as a kind of important neural networks, studies have shown that the fuzzy neural networks are a very useful paradigm for image processing problems [17] [18]. Subsequently, various interesting results on the stability and other behaviors of delayed fuzzy BAM neural networks have been derived (see [19] [20] [21] [22] and references cited therein). However, to the best of our knowledge, there are few results on the uniform stability analysis of fractional-order fuzzy BAM neural networks with leakage delays.

Motivated by the above, in this paper, we are concerned with the following fractional-order fuzzy BAM neural network with delays in the leakage terms:

$$
\left\{\begin{aligned}
{ }^{c} D^{\alpha} x_{i}(t) & =-a_{i} x_{i}(t-\sigma)+\sum_{j=1}^{m} c_{j i} f_{j}\left(y_{j}(t-\tau)\right)+\bigwedge_{j=1}^{m} \alpha_{j i} f_{j}\left(y_{j}(t-\tau)\right) \\
& +\bigvee_{j=1}^{m} \beta_{j i} f_{j}\left(y_{j}(t-\tau)\right)+\bigwedge_{j=1}^{m} T_{j i} \mu_{j}+\bigvee_{j=1}^{m} H_{j i} \mu_{j}+I_{i}, i=1,2, \cdots, n, t \geq 0, \\
{ }^{c} D^{\alpha} y_{j}(t) & =-b_{j} y_{j}(t-\tau)+\sum_{i=1}^{n} d_{i j} g_{i}\left(x_{i}(t-\sigma)\right)+\bigwedge_{i=1}^{n} p_{i j} g_{i}\left(x_{i}(t-\sigma)\right) \\
& +\bigvee_{i=1}^{n} q_{i j} g_{i}\left(x_{i}(t-\sigma)\right)+\bigwedge_{i=1}^{n} K_{i j} v_{i}+\bigvee_{i=1}^{n} R_{i j} v_{i}+J_{j}, j=1,2, \cdots, m, t \geq 0,
\end{aligned}\right.
$$

where $n$ and $m$ correspond to the number of neurons in X-layer and Y-layer, respectively. ${ }^{c} D^{\alpha}$ is the Caputo's fractional derivative and $0<\alpha<1 ; x_{i}(t)$ and $y_{j}(t)$ are the activations of the $i$ th neuron and the $j$ theuron, respectively; $a_{i}>0, b_{j}>0$ denote the rate with which the $i$ th neurons and the $j$ th neurons will reset its potential to the resting state in isolation when disconnected from 
the network and external inputs; $a_{i j}, b_{i j}, T_{i j}$ and $H_{i j}$ are the elements of fuzzy feedback MIN template and fuzzy feedback MAX template, fuzzy feed-forward MIN template and fuzzy feed-forward MAX template in X-layer, respectively; $p_{i j}, q_{i j}, K_{i j}$ and $R_{i j}$ are the elements of fuzzy feedback MIN template and fuzzy feedback MAX template, fuzzy feed-forward MIN template and fuzzy feed-forward MAX template in Y-layer, respectively; $\wedge$ and $\vee$ denote the fuzzy AND and fuzzy OR operation, respectively; $\mu_{j}$ and $v_{i}$ denote external input of the $i$ th neurons in X-layer and external input of the $t$ th neurons in Y-layer, respectively; $I_{i}$ and $J_{j}$ represent bias of the ith neurons in X-layer and bias of the th neurons in Y-layer, respectively; $c_{i j}$ and $d_{i j}$ are the delayed feedback, $f_{j}(\cdot)$ and $g_{j}(\cdot)$ are the signal transmission functions, and $i=1,2, \cdots, n ; j=1,2, \cdots, m$.

Here, the initial conditions associated with system (1) are of the form

$$
\left\{\begin{array}{l}
x_{i}(s)=\phi_{i}(s), \phi_{i}(0)=0, s \in[-\sigma, 0], i=1,2, \cdots, n, \\
y_{j}(s)=\varphi_{j}(s), \varphi_{j}(0)=0, s \in[-\tau, 0], j=1,2, \cdots, m,
\end{array}\right.
$$

where it is usually assumed that $\phi_{i}(\cdot)$ and $\varphi_{j}(\cdot)$ denote real-valued continuous functions defined on $[-\sigma, 0]$ and $[-\tau, 0]$, respectively. If the initial value

$$
\psi(t)=\left(\phi_{1}(t), \phi_{2}(t), \cdots, \phi_{n}(t), \varphi_{1}(t), \varphi_{2}(t), \cdots, \varphi_{m}(t)\right)^{\mathrm{T}},
$$

we denote the norm $\|\psi\|=\max \left\{\|\phi\|_{0},\|\varphi\|_{0}\right\}$, norm of $\|\cdot\|_{0}$ denoted by

$$
\|\phi\|_{0}=\sum_{i=1}^{n} \sup _{t \in[-\sigma, 0]}\left\{\mathrm{e}^{-t}\left|\phi_{i}(t)\right|\right\},\|\varphi\|_{0}=\sum_{j=1}^{m} \sup _{t \in[-\tau, 0]}\left\{\mathrm{e}^{-t}\left|\varphi_{j}(t)\right|\right\} .
$$

The main purpose of this paper is to obtain some sufficient conditions for the uniform stability of the system. Then we study the existence, uniqueness, uniform stability of the equilibrium point.

This paper is organized as follows: In Section 2, we introduce some notations and definitions and state some preliminary results which are needed in later sections. In Section 3, we establish some sufficient conditions for the uniform stability of the system and the existence, uniqueness, and uniform stability of the equilibrium point. In Section 4, an example is given to illustrate that our results are feasible. The conclusion is made in Section 5.

\section{Preliminaries}

In this section, we shall recall some definitions and state some lemmas which will be used in the later section.

Definition 1. ([1], [2]) The fractional integral (Riemann-Liouville integral) $I_{t_{0}, t}^{\alpha}$ with fractional order $\alpha \in R^{+}$of function $x(t)$ is defined as

$$
I_{t_{0}, t}^{\alpha} x(t)=\frac{1}{\Gamma(\alpha)} \int_{t_{0}}^{t}(t-\tau)^{\alpha-1} x(\tau) \mathrm{d} \tau,
$$

where $\Gamma(\cdot)$ is the gamma function, $\Gamma(\alpha)=\int_{0}^{+\infty} t^{\alpha-1} \mathrm{e}^{-t} \mathrm{~d} t$. 
Definition 2. ([1], [2]) The Riemann-Liouville derivative of fractional order $\alpha$ of function $x(t)$ is given as

$$
D_{t_{0}, t}^{\alpha} x(t)=\frac{\mathrm{d}^{n}}{\mathrm{~d} t^{n}} \frac{1}{\Gamma(n-\alpha)} \int_{t_{0}}^{t}(t-\tau)^{n-\alpha-1} x(\tau) \mathrm{d} \tau,
$$

where $n-1<\alpha<n \in Z^{+}$.

Definition 3. ([1], [2]) The Caputo derivative of fractional order $\alpha$ of function $x(t)$ is defined as follows

$$
{ }^{c} D_{t_{0}, t}^{\alpha} x(t)=\frac{1}{\Gamma(n-\alpha)} \int_{t_{0}}^{t} \frac{x^{(n)}(\tau)}{(t-\tau)^{\alpha-n+1}} \mathrm{~d} \tau,
$$

where $n-1<\alpha<n \in Z^{+}$.

Lemma 1. ([1], [2]) If $x(t) \in C^{n}[0,+\infty)$ and $n-1<\alpha<n \in Z^{+}$, then

1) $I^{\alpha} I^{\beta} x(t)=I^{\alpha+\beta} x(t), \alpha, \beta \geq 0$;

2) ${ }^{c} D^{\alpha} I^{\beta} x(t)=x(t), \alpha=\beta$;

3) $I^{\alpha c} D^{\beta} x(t)=x(t)-\sum_{k=0}^{n-1} \frac{t^{k}}{k !} x\left(t_{0}\right), \alpha=\beta \geq 0$.

Consider the initial value problem of the following fractional differential equation

$$
\left\{\begin{array}{l}
{ }^{c} D^{\alpha} x(t)=f(t, x(t)), t \in[0,+\infty), \\
x(0)=x_{0}, x_{0} \in R^{m}
\end{array}\right.
$$

where $\quad x=\left(x_{1}, x_{2}, \cdots, x_{m}\right)^{\mathrm{T}} \in R^{m}, \quad 0<\alpha<1, \quad f:[0,+\infty) \times R^{m} \rightarrow R^{m} \quad$ is continuous in $t$ and locally Lipschitz in $x$.

The equilibrium point of the Caputo's fractional dynamic system has been defined in earlier work [23] [24]. We shall employ the following definitions of the equilibrium point and uniformly stable of the Caputo's fractional dynamic system:

Definition 3. The constant $x^{*}$ is an equilibrium point of the Caputo's fractional dynamic system (3) if and only if $f\left(t, x^{*}\right)=0$ for any $t \in[0,+\infty)$.

Definition 4. The solution of system (1) is said to be stable if for any $\varepsilon>0$ there exists $\delta\left(t_{0}, \varepsilon\right)>0$ such that $t \geq t_{0} \geq 0,\left\|x_{0}-y_{0}\right\|<\delta$ imply $\left\|y\left(t, t_{0}, y_{0}\right)-x\left(t, t_{0}, x_{0}\right)\right\|<\varepsilon$ for any two solutions $x\left(t, t_{0}, x_{0}\right)$ and $y\left(t, t_{0}, y_{0}\right)$. It is uniformly stable if the above $\delta$ independent of $t_{0}$.

Definition 5. The solution $z^{*}(t)=\left(x_{1}^{*}(t), x_{2}^{*}(t), \cdots, x_{n}^{*}(t), y_{1}^{*}(t), y_{2}^{*}(t), \cdots, y_{m}^{*}(t)\right)^{\mathrm{T}} \quad$ with initial values $\psi^{*}(t)=\left(\phi_{1}^{*}(t), \phi_{2}^{*}(t), \cdots, \phi_{n}^{*}(t), \varphi_{1}^{*}(t), \varphi_{2}^{*}(t), \cdots, \varphi_{m}^{*}(t)\right)^{\mathrm{T}}$ of system (1) is said to be uniformly stable if for any $\varepsilon>0$ and $\delta(\varepsilon)>0$ for any solution

$$
z(t)=\left(x_{1}(t), x_{2}(t), \cdots, x_{n}(t), y_{1}(t), y_{2}(t), \cdots, y_{m}(t)\right)^{\mathrm{T}}
$$

of (1) with initial value $\psi(t)=\left(\phi_{1}(t), \phi_{2}(t), \cdots, \phi_{n}(t), \varphi_{1}(t), \varphi_{2}(t), \cdots, \varphi_{m}(t)\right)^{\mathrm{T}}$, where

$$
\left(\phi_{1}, \phi_{2}, \cdots, \phi_{n}\right) \in C\left([-\sigma, 0], R^{n}\right),\left(\varphi_{1}, \varphi_{2}, \cdots, \varphi_{m}\right), \in C\left([-\tau, 0], R^{m}\right)
$$


and

$$
\phi_{i}(0)=0, \varphi_{j}(0)=0, i=1,2, \cdots, n ; j=1,2, \cdots, m,
$$

such that $\left\|\psi^{*}\left(t_{0}\right)-\psi\left(t_{0}\right)\right\|<\delta$ imply $\left\|z^{*}\left(t, t_{0}, \psi^{*}\left(t_{0}\right)\right)-z\left(t, t_{0}, \psi\left(t_{0}\right)\right)\right\|<\varepsilon$.

In order to obtain the main results, here, we make the following assumptions:

$\left(\mathrm{H}_{1}\right)$ The neuron activation functions $f_{j}, g_{i} \in C[R, R]$ $(i=1,2, \cdots, n, j=1,2, \cdots, m)$ satisfy the Lipschitz condition. That is, there exist positive constants $F_{j}, G_{i}$ such that

$$
\left|f_{j}(u)-f_{j}(v)\right|<F_{j}|u-v|,\left|g_{i}(u)-g_{i}(v)\right|<G_{i}|u-v|, \forall u, v \in R
$$

Lemma 2. ([25]) Suppose that $x, y \in R^{n}$ be the two states of the system (1). Then, one has

$$
\begin{aligned}
& \left|\bigwedge_{j=1}^{n} \alpha_{i j} f_{j}\left(x_{j}\right)-\bigwedge_{j=1}^{n} \alpha_{i j} f_{j}\left(y_{j}\right)\right| \leq \sum_{j=1}^{n}\left|\alpha_{i j}\right|\left|f_{j}\left(x_{j}\right)-f_{j}\left(y_{j}\right)\right|, \\
& \left|\bigvee_{j=1}^{n} \beta_{i j} f_{j}\left(x_{j}\right)-\bigvee_{j=1}^{n} \beta_{i j} f_{j}\left(y_{j}\right)\right| \leq \sum_{j=1}^{n}\left|\beta_{i j}\right|\left|f_{j}\left(x_{j}\right)-f_{j}\left(y_{j}\right)\right| .
\end{aligned}
$$

\section{Uniform Stability of Fractional-Order Neural Networks}

In this section, a sufficient condition for uniform stability of a class of fractional-order delayed neural networks on time scale, and the existence and uniqueness, uniform stability of equilibrium point are proposed, respectively.

Theorem 3. Let $\left(\mathrm{H}_{1}\right)$ holds. Suppose further that, for $i=1,2, \cdots, n ; j=1,2, \cdots, m$, $\left(\mathrm{H}_{2}\right) a_{i}, b_{j}, c_{j i}, \alpha_{j i}, \beta_{j i}, d_{i j}, p_{i j}, q_{i j}, F_{j}$ and $G_{i}$ satisfy the following condition: $C>K$, where

$$
\begin{gathered}
w_{j i}=\left|c_{j i}\right|+\left|\alpha_{j i}\right|+\left|\beta_{j i}\right|, A=\sum_{i=1}^{n} \omega_{i}^{*}=\sum_{i=1}^{n} \max _{\forall j}\left\{w_{j i} F_{j}\right\}, \\
\vartheta_{i j}=\left|d_{i j}\right|+\left|p_{i j}\right|+\left|q_{i j}\right|, B=\sum_{j=1}^{m} \vartheta_{j}^{*}=\sum_{j=1}^{m} \max _{\forall i}\left\{\vartheta_{i j} G_{i}\right\}, \\
\bar{a}=\max _{\forall i}\left\{a_{i}\right\}, \bar{b}=\max _{\forall j}\left\{b_{j}\right\}, \underline{a}=\max _{\forall i}\left\{a_{i}\right\}, \underline{b}=\max _{\forall j}\left\{b_{j}\right\}, \\
C=\min \{1-\bar{a}, \underline{a}, 1-\bar{b}, \underline{b}\}, K=\max \left\{(\bar{b}+A) \mathrm{e}^{-\sigma},(\bar{a}+B) \mathrm{e}^{-\tau}\right\},
\end{gathered}
$$

then the system (1) is uniformly stable.

Proof. Assume that

$$
z(t)=\left(x_{1}(t), x_{2}(t), \cdots, x_{n}(t), y_{1}(t), y_{2}(t), \cdots, y_{m}(t)\right)^{\mathrm{T}}
$$

and

$$
z^{*}(t)=\left(x_{1}^{*}(t), x_{2}^{*}(t), \cdots, x_{n}^{*}(t), y_{1}^{*}(t), y_{2}^{*}(t), \cdots, y_{m}^{*}(t)\right)^{\mathrm{T}}
$$

are two solutions of system (1) with different initial values

$$
\begin{gathered}
\psi(t)=\left(\phi_{1}(t), \phi_{2}(t), \cdots, \phi_{n}(t), \varphi_{1}(t), \varphi_{2}(t), \cdots, \varphi_{m}(t)\right)^{\mathrm{T}}, \\
\psi^{*}(t)=\left(\phi_{1}^{*}(t), \phi_{2}^{*}(t), \cdots, \phi_{n}^{*}(t), \varphi_{1}^{*}(t), \varphi_{2}^{*}(t), \cdots, \varphi_{m}^{*}(t)\right)^{\mathrm{T}},
\end{gathered}
$$

where 


$$
\begin{aligned}
& \left(\phi_{1}, \phi_{2}, \cdots, \phi_{n}\right),\left(\phi_{1}^{*}, \phi_{2}^{*}, \cdots, \phi_{n}^{*}\right) \in C\left([-\sigma, 0], R^{n}\right), \\
& \left(\varphi_{1}, \varphi_{2}, \cdots, \varphi_{m}\right),\left(\varphi_{1}^{*}, \varphi_{2}^{*}, \cdots, \varphi_{m}^{*}\right) \in C\left([-\tau, 0], R^{m}\right)
\end{aligned}
$$

and

$$
\phi_{i}(0)=0, \phi_{i}^{*}(0)=0, i=1,2, \cdots, n ; \quad \varphi_{j}(0)=0, \varphi_{j}^{*}(0)=0, j=1,2, \cdots, m .
$$

If $z(t)=\left(x_{1}(t), x_{2}(t), \cdots, x_{n}(t), y_{1}(t), y_{2}(t), \cdots, y_{m}(t)\right)^{\mathrm{T}}$, we use the following norm:

$$
\|z\|=\max \left\{\|x\|_{0},\|y\|_{0}\right\},
$$

norm of $\|.\|_{0}$ denoted by

$$
\|x\|_{0}=\sum_{i=1}^{n} \sup _{t \in R}\left\{\mathrm{e}^{-t}\left|x_{i}(t)\right|\right\},\|y\|_{0}=\sum_{j=1}^{m} \sup _{t \in R}\left\{\mathrm{e}^{-t}\left|y_{j}(t)\right|\right\} .
$$

Based on Lemma 2, one has

$$
\begin{aligned}
& \mathrm{e}^{-t}\left|x_{i}(t)-x_{i}^{*}(t)\right| \\
& \leq \frac{1}{\Gamma(\alpha)} \mathrm{e}^{-t} \int_{0}^{t}(t-s)^{\alpha-1}\left\{a_{i}\left|x_{i}(s-\sigma)-x_{i}^{*}(s-\sigma)\right|\right. \\
& \left.+\sum_{j=1}^{m}\left(\left|c_{j i}\right|+\left|\alpha_{j i}\right|+\left|\beta_{j i}\right|\right)\left|f_{j}\left(y_{j}(s-\tau)\right)-f_{j}\left(y_{j}^{*}(s-\tau)\right)\right|\right\} \mathrm{d} s \\
& \leq a_{i} \frac{1}{\Gamma(\alpha)} \int_{0}^{\sigma}(t-s)^{\alpha-1} \mathrm{e}^{-(t-s)} \mathrm{e}^{-s}\left|\phi_{i}(s-\sigma)-\phi_{i}^{*}(s-\sigma)\right| \mathrm{d} s \\
& +a_{i} \frac{1}{\Gamma(\alpha)} \int_{\sigma}^{t}(t-s)^{\alpha-1} \mathrm{e}^{-(t-s)} \mathrm{e}^{-s}\left|x_{i}(s-\sigma)-x_{i}^{*}(s-\sigma)\right| \mathrm{d} s \\
& +\sum_{j=1}^{m} w_{j i} F_{j} \frac{1}{\Gamma(\alpha)} \int_{0}^{\tau}(t-s)^{\alpha-1} \mathrm{e}^{-(t-s)} \mathrm{e}^{-s}\left|\varphi_{j}(s-\tau)-\varphi_{j}^{*}(s-\tau)\right| \mathrm{d} s \\
& +\sum_{j=1}^{m} w_{j i} F_{j} \frac{1}{\Gamma(\alpha)} \int_{\tau}^{t}(t-s)^{\alpha-1} \mathrm{e}^{-(t-s)} \mathrm{e}^{-s}\left|y_{j}(s-\tau)-y_{j}^{*}(s-\tau)\right| \mathrm{d} s \\
& \leq a_{i} \frac{1}{\Gamma(\alpha)} \int_{-\sigma}^{0}(t-\zeta-\sigma)^{\alpha-1} \mathrm{e}^{-(t-\zeta)} \mathrm{e}^{-\zeta}\left|\phi_{i}(\zeta)-\phi_{i}^{*}(\zeta)\right| \mathrm{d} \zeta \\
& +a_{i} \frac{1}{\Gamma(\alpha)} \int_{0}^{t-\sigma}(t-\zeta-\sigma)^{\alpha-1} \mathrm{e}^{-(t-\zeta)} e^{-\zeta}\left|x_{i}(\zeta)-x_{i}^{*}(\zeta)\right| \mathrm{d} \zeta \\
& +w_{i}^{*} \sum_{j=1}^{m} \frac{1}{\Gamma(\alpha)} \int_{-\tau}^{0}(t-\gamma-\tau)^{\alpha-1} \mathrm{e}^{-(t-\gamma)} \mathrm{e}^{-\gamma}\left|\varphi_{j}(\gamma)-\varphi_{j}^{*}(\gamma)\right| \mathrm{d} \gamma \\
& +w_{i}^{*} \sum_{j=1}^{m} \frac{1}{\Gamma(\alpha)} \int_{0}^{t-\tau}(t-\gamma-\tau)^{\alpha-1} \mathrm{e}^{-(t-\gamma)} \mathrm{e}^{-\gamma}\left|y_{j}(\gamma)-y_{j}^{*}(\gamma)\right| \mathrm{d} \gamma \\
& \leq a_{i} \sup _{t \in \mathbb{R}}\left\{\mathrm{e}^{-t}\left|\phi_{i}(t)-\phi_{i}^{*}(t)\right|\right\} \mathrm{e}^{-\sigma} \frac{1}{\Gamma(\alpha)} \int_{t-\sigma}^{t} \eta^{\alpha-1} \mathrm{e}^{-\eta} \mathrm{d} \eta \\
& +a_{i} \sup _{t \in \mathbb{R}}\left\{\mathrm{e}^{-t}\left|x_{i}(t)-x_{i}^{*}(t)\right|\right\} \mathrm{e}^{-\sigma} \frac{1}{\Gamma(\alpha)} \int_{0}^{t-\sigma} \eta^{\alpha-1} \mathrm{e}^{-\eta} \mathrm{d} \eta \\
& +w_{i}^{*} \sum_{j=1}^{m} \sup _{t \in \mathbb{R}}\left\{\mathrm{e}^{-t}\left|\varphi_{j}(t)-\varphi_{j}^{*}(t)\right|\right\} \mathrm{e}^{-\tau} \frac{1}{\Gamma(\alpha)} \int_{t-\tau}^{t} \theta^{\alpha-1} \mathrm{e}^{-\theta} \mathrm{d} \theta \\
& +w_{i}^{*} \sum_{j=1}^{n} \sup _{t \in \mathbb{R}}\left\{\mathrm{e}^{-t}\left|y_{j}(t)-y_{j}^{*}(t)\right|\right\} \mathrm{e}^{-\tau} \frac{1}{\Gamma(\alpha)} \int_{0}^{t-\tau} \theta^{\alpha-1} \mathrm{e}^{-\theta} \mathrm{d} \theta
\end{aligned}
$$




$$
\begin{aligned}
\leq & a_{i} \sup _{t \in \mathbb{R}}\left\{\mathrm{e}^{-t}\left|\phi_{i}(t)-\phi_{i}^{*}(t)\right|\right\} \mathrm{e}^{-\sigma}+a_{i} \sup _{t \in \mathbb{R}}\left\{\mathrm{e}^{-t}\left|x_{i}(t)-x_{i}^{*}(t)\right|\right\} \mathrm{e}^{-\sigma} \\
& +w_{i}^{*} \sum_{j=1}^{m} \sup _{t \in \mathbb{R}}\left\{\mathrm{e}^{-t}\left|\varphi_{j}(t)-\varphi_{j}^{*}(t)\right|\right\} \mathrm{e}^{-\tau}+w_{i}^{*} \sum_{j=1}^{m} \sup _{t \in \mathbb{R}}\left\{\mathrm{e}^{-t}\left|y_{i}(t)-y_{i}^{*}(t)\right|\right\} \mathrm{e}^{-\tau} \\
\leq & a_{i} e^{-\sigma}\left\|\phi-\phi^{*}\right\|_{0}+a_{i} \mathrm{e}^{-\sigma}\left\|x-x^{*}\right\|_{0} \\
& +w_{i}^{*} \mathrm{e}^{-\tau}\left\|y-y^{*}\right\|_{0}+w_{i}^{*} \mathrm{e}^{-\tau}\left\|\varphi-\varphi^{*}\right\|_{0}, \quad i=1,2, \cdots, n .
\end{aligned}
$$

From (4), one obtains

$$
\begin{aligned}
\left\|x-x^{*}\right\|_{0} & =\sum_{i=1}^{n} \sup _{t \in \mathbb{R}}\left\{\mathrm{e}^{-t}\left|x_{i}(t)-x_{i}^{*}(t)\right|\right\} \\
& \leq \bar{a} \mathrm{e}^{-\sigma}\left(\left\|\phi-\phi^{*}\right\|_{0}+\left\|x-x^{*}\right\|_{0}\right)+A \mathrm{e}^{-\tau}\left(\left\|y-y^{*}\right\|_{0}+\left\|\varphi-\varphi^{*}\right\|_{0}\right),
\end{aligned}
$$

which implies that

$$
\left(1-\bar{a} \mathrm{e}^{-\sigma}\right)\left\|x-x^{*}\right\|_{0} \leq \bar{a} \mathrm{e}^{-\sigma}\left\|\phi-\phi^{*}\right\|_{0}+A \mathrm{e}^{-\tau}\left(\left\|y-y^{*}\right\|_{0}+\left\|\varphi-\varphi^{*}\right\|_{0}\right) .
$$

Similarly, we can also get

$$
\left(1-\bar{b} \mathrm{e}^{-\tau}\right)\left\|y-y^{*}\right\|_{0} \leq \bar{b} \mathrm{e}^{-\tau}\left\|\varphi-\varphi^{*}\right\|_{0}+B \mathrm{e}^{-\sigma}\left(\left\|x-x^{*}\right\|_{0}+\left\|\phi-\phi^{*}\right\|_{0}\right) .
$$

In view of (5) and (6), one has

$$
\begin{aligned}
& C\left(\left\|x-x^{*}\right\|_{0}+\left\|y-y^{*}\right\|_{0}\right) \\
& \leq K\left(\left\|x-x^{*}\right\|_{0}+\left\|y-y^{*}\right\|_{0}\right)+K\left(\left\|\phi-\phi^{*}\right\|_{0}+\left\|\varphi-\varphi^{*}\right\|_{0}\right),
\end{aligned}
$$

which implies that

$$
\left\|x-x^{*}\right\|_{0}+\left\|y-y^{*}\right\|_{0} \leq \frac{K}{C-K}\left(\left\|\phi-\phi^{*}\right\|_{0}+\left\|\varphi-\varphi^{*}\right\|_{0}\right) .
$$

Easily, we have

$$
\left\|z-z^{*}\right\| \leq \frac{K}{C-K}\left\|\psi-\psi^{*}\right\| .
$$

Therefore, for $\forall \varepsilon>0$, there exist $\delta=(C-K) \varepsilon / K>0$ such that $\left\|z-z^{*}\right\|<\varepsilon$ when $\left\|\psi-\psi^{*}\right\|<\delta$, which means that the solution $z(t)$ the system (1) is uniformly stable.

Theorem 4. Let $\left(\mathrm{H}_{1}\right),\left(\mathrm{H}_{2}\right)$ hold, then there exists a unique equilibrium point in system (1), which is uniformly stable.

Proof: Let $a_{i} x_{i}^{*}=u_{i}^{*}, b_{j} y_{j}^{*}=v_{j}^{*}$, and constructing a mapping $\Theta: R^{n+m} \rightarrow R^{n+m}$ defined by

$$
\Theta(z)=\left(\Phi_{1} u_{1}, \cdots, \Phi_{n} u_{n}, \Psi_{1} v_{1}, \cdots, \Psi_{m} v_{m}\right)^{\mathrm{T}},
$$

where

$$
\begin{gathered}
z=\left(u_{1}, u_{2}, \cdots, u_{n}, v_{1}, v_{2}, \cdots, v_{m}\right)^{\mathrm{T}}, \\
\Phi_{i} u_{i}=\sum_{j=1}^{m} c_{j i} f_{j}\left(\frac{v_{j}^{*}}{b_{j}}\right)+\bigwedge_{j=1}^{m} \alpha_{j i} f_{j}\left(\frac{v_{j}^{*}}{b_{j}}\right)+\bigvee_{j=1}^{m} \beta_{j i} f_{j}\left(\frac{v_{j}^{*}}{b_{j}}\right) \\
+\bigwedge_{j=1}^{m} T_{j i} \mu_{j}+\bigvee_{j=1}^{m} H_{j i} \mu_{j}+I_{i}, i=1,2, \cdots, n, t \geq 0,
\end{gathered}
$$




$$
\begin{aligned}
\Psi_{j} v_{j}= & \sum_{i=1}^{n} d_{i j} g_{i}\left(\frac{u_{i}^{*}}{c_{i}}\right)+\bigwedge_{i=1}^{n} p_{i j} g_{i}\left(\frac{u_{i}^{*}}{c_{i}}\right)+\bigvee_{i=1}^{n} q_{i j} g_{i}\left(\frac{u_{i}^{*}}{c_{i}}\right) \\
& +\bigwedge_{i=1}^{n} K_{i j} v_{i}+\bigvee_{i=1}^{n} R_{i j} v_{i}+J_{j}, j=1,2, \cdots, m, t \geq 0
\end{aligned}
$$

Now, we will show that $\Theta$ is a contraction mapping on $R^{n+m}$ endowed with the norm

$$
\|z\|=\max \left\{\sum_{i=1}^{n}\left|u_{i}\right|, \sum_{j=1}^{m}\left|v_{j}\right|\right\} .
$$

In fact, for any two different points

$$
z=\left(u_{1}, u_{2}, \cdots, u_{n}, v_{1}, v_{2}, \cdots, v_{m}\right)^{\mathrm{T}}
$$

and

$$
\bar{z}=\left(\bar{u}_{1}, \bar{u}_{2}, \cdots, \bar{u}_{n}, \bar{v}_{1}, \bar{v}_{2}, \cdots, \bar{v}_{m}\right)^{\mathrm{T}},
$$

we have

$$
\begin{aligned}
& \|\Theta(z)-\Theta(\bar{z})\| \\
& =\max \left\{\sum_{i=1}^{n}\left|\Phi_{i} u_{i}-\Phi_{i} \overline{u_{i}}\right|, \sum_{j=1}^{m}\left|\Psi_{j} v_{j}-\Psi_{j} \overline{v_{j}}\right|\right\} \\
& \leq \max \left\{\sum _ { i = 1 } ^ { n } \left(\sum_{j=1}^{m}\left|c_{j i}\right|\left|f_{j}\left(\frac{v_{j}}{b_{j}}\right)-f_{j}\left(\frac{\overline{v_{j}}}{b_{j}}\right)\right|+\sum_{j=1}^{m}\left|\alpha_{j i}\right|\left|f_{j}\left(\frac{v_{j}}{b_{j}}\right)-f_{j}\left(\frac{\overline{v_{j}}}{b_{j}}\right)\right|\right.\right. \\
& \left.+\sum_{j=1}^{m}\left|\beta_{j i}\right|\left|f_{j}\left(\frac{v_{j}}{b_{j}}\right)-f_{j}\left(\frac{\overline{v_{j}}}{b_{j}}\right)\right|\right), \sum_{j=1}^{m}\left(\sum_{i=1}^{n}\left|d_{i j}\right|\left|g_{i}\left(\frac{u_{i}}{c_{i}}\right)-\left(\frac{\overline{u_{i}}}{c_{i}}\right)\right|\right. \\
& \left.\left.+\sum_{i=1}^{n}\left|p_{i j}\right|\left|g_{i}\left(\frac{u_{i}}{c_{i}}\right)-\left(\frac{\overline{u_{i}}}{c_{i}}\right)\right|+\sum_{i=1}^{n}\left|q_{i j}\right|\left|g_{i}\left(\frac{u_{i}}{c_{i}}\right)-\left(\frac{\overline{u_{i}}}{c_{i}}\right)\right|\right)\right\} \\
& \leq \max \left\{\sum_{i=1}^{n} \sum_{j=1}^{m} \frac{w_{j i} F_{j}}{b_{j}}\left|v_{j}-\overline{v_{j}}\right|, \sum_{j=1}^{m} \sum_{i=1}^{n} \frac{\vartheta_{i j} G_{i}}{a_{i}}\left|u_{i}-\overline{u_{i}}\right|\right\} \\
& \leq \max \left\{\sum_{i=1}^{n} \frac{w_{i}^{*}}{w_{j}^{*}} \sum_{j=1}^{m}\left|v_{j}(t)-\overline{v_{j}}\right|, \sum_{j=1}^{m} \frac{\vartheta_{i}^{*}}{\underline{a}} \sum_{i=1}^{n}\left|u_{i}-\overline{u_{i}}\right|\right\} \\
& \leq \max \left\{\frac{A}{C} \sum_{j=1}^{m}\left|v_{j}-\overline{v_{j}}\right|, \frac{B}{C} \sum_{i=1}^{n}\left|u_{i}-\overline{u_{i}}\right|\right\} \\
& \leq \frac{K}{C} \max \left\{\sum_{j=1}^{m}\left|v_{j}-\overline{v_{j}}\right|, \sum_{i=1}^{n}\left|u_{i}-\overline{u_{i}}\right|\right\} \\
& \leq \frac{K}{C}\|z-\bar{z}\| .
\end{aligned}
$$

It follows from $\left(\mathrm{H}_{2}\right)$ that

$$
\|\Theta(z)-\Theta(\bar{z})\|<\|z-\bar{z}\|,
$$

which implies that $\Theta$ is a contraction mapping on $R^{n+m}$. Hence, there exists a unique fixed point $u^{*}$ such that $\Theta\left(z^{*}\right)=z^{*}=\left(u_{1}^{*}, u_{2}^{*}, \cdots, u_{n}^{*}, v_{1}^{*}, v_{2}^{*}, \cdots, v_{m}^{*}\right)^{\mathrm{T}}$, i.e. 


$$
\left\{\begin{aligned}
u_{i}^{*}= & \sum_{j=1}^{m} c_{j i} f_{j}\left(\frac{v_{j}^{*}}{b_{j}}\right)+\bigwedge_{j=1}^{m} \alpha_{j i} f_{j}\left(\frac{v_{j}^{*}}{b_{j}}\right)+\bigvee_{j=1}^{m} \beta_{j i} f_{j}\left(\frac{v_{j}^{*}}{b_{j}}\right) \\
& +\bigwedge_{j=1}^{m} T_{j i} \mu_{j}+\bigvee_{j=1}^{m} H_{j i} \mu_{j}+I_{i}, \quad i=1,2, \cdots, n, t \geq 0, \\
v_{j}^{*}= & \sum_{i=1}^{n} d_{i j} g_{i}\left(\frac{u_{i}^{*}}{c_{i}}\right)+\bigwedge_{i=1}^{n} p_{i j} g_{i}\left(\frac{u_{i}^{*}}{c_{i}}\right)+\bigvee_{i=1}^{n} q_{i j} g_{i}\left(\frac{u_{i}^{*}}{c_{i}}\right) \\
& +\bigwedge_{i=1}^{n} K_{i j} v_{i}+\bigvee_{i=1}^{n} R_{i j} v_{i}+J_{j}, \quad j=1,2, \cdots, m, t \geq 0 .
\end{aligned}\right.
$$

That is

$$
\left\{\begin{aligned}
-a_{i} x_{i}^{*} & +\sum_{j=1}^{m} c_{j i} f_{j}\left(y_{j}^{*}\right)+\bigwedge_{j=1}^{m} \alpha_{j i} f_{j}\left(y_{j}^{*}\right)+\bigvee_{j=1}^{m} \beta_{j i} f_{j}\left(y_{j}^{*}\right) \\
& +\bigwedge_{j=1}^{m} T_{j i} \mu_{j}+\bigvee_{j=1}^{m} H_{j i} \mu_{j}+I_{i}=0, i=1,2, \cdots, n, t \geq 0 \\
-b_{j} y_{j}^{*} & +\sum_{i=1}^{n} d_{i j} g_{i}\left(x_{i}^{*}\right)+\bigwedge_{i=1}^{n} p_{i j} g_{i}\left(x_{i}^{*}\right)+\bigvee_{i=1}^{n} q_{i j} g_{i}\left(x_{i}^{*}\right) \\
& +\bigwedge_{i=1}^{n} K_{i j} v_{i}+\bigvee_{i=1}^{n} R_{i j} v_{i}+J_{j}=0, j=1,2, \cdots, m, t \geq 0
\end{aligned}\right.
$$

which implies that $x^{*}$ is an equilibrium point of the system (1). Moreover, it follows from Theorem 3 that $x^{*}$ is uniformly stable. This completes the proof.

\section{Numerical Example}

In this section, a numerical example is presented to illustrate our results. Consider the following fractional-order fuzzy BAM neural network:

$$
\left\{\begin{aligned}
{ }^{c} D^{\alpha} x_{i}(t)= & -a_{i} x_{i}(t-\sigma)+\sum_{j=1}^{m} c_{j i} f_{j}\left(y_{j}(t-\tau)\right)+\bigwedge_{j=1}^{m} \alpha_{j i} f_{j}\left(y_{j}(t-\tau)\right) \\
& +\bigvee_{j=1}^{m} \beta_{j i} f_{j}\left(y_{j}(t-\tau)\right)+\bigwedge_{j=1}^{m} T_{j i} \mu_{j}+\bigvee_{j=1}^{m} H_{j i} \mu_{j}+I_{i}, i=1,2, \cdots, n, t \geq 0, \\
{ }^{c} D^{\alpha} y_{j}(t) & =-b_{j} y_{j}(t-\tau)+\sum_{i=1}^{n} d_{i j} g_{i}\left(x_{i}(t-\sigma)\right)+\bigwedge_{i=1}^{n} p_{i j} g_{i}\left(x_{i}(t-\sigma)\right) \\
& +\bigvee_{i=1}^{n} q_{i j} g_{i}\left(x_{i}(t-\sigma)\right)+\bigwedge_{i=1}^{n} K_{i j} v_{i}+\bigvee_{i=1}^{n} R_{i j} v_{i}+J_{j}, j=1,2, \cdots, m, t \geq 0,
\end{aligned}\right.
$$

where

$$
\begin{gathered}
F_{j}=\frac{1}{2}, G_{i}=\frac{1}{5}, \tau=\sigma=1, m=n=1,2, \\
f_{j}\left(y_{j}(t-\tau)\right)=\frac{1}{2} \sin \left(y_{j}(t-\tau)\right), g_{i}\left(x_{i}(t-\sigma)\right)=\frac{1}{5} \cos \left(x_{i}(t-\sigma)\right), \\
a_{1}=0.45 ; a_{2}=0.4 ; b_{1}=0.5 ; b_{2}=0.4 ; c_{11}=c_{12}=c_{21}=c_{22}=0.01 ; \\
d_{11}=d_{12}=0.02 ; d_{21}=d_{22}=0.03 ; \alpha_{11}=\alpha_{12}=0.02 ; \alpha_{21}=\alpha_{22}=0.02 ; \\
\beta_{11}=\beta_{12}=0.05 ; \beta_{21}=\beta_{22}=0.02 ; p_{11}=p_{12}=0.03 ; p_{21}=p_{22}=0.04 ; \\
q_{11}=q_{12}=0.01 ; q_{21}=q_{22}=0.03 ; T_{j i}=H_{j i}=K_{i j}=R_{i j}=1 ; \\
\mu_{j}=v_{i}=1 ; I_{i}=J_{j}=2 .
\end{gathered}
$$


When $t \geq 0$, we have

$$
\bar{a}=0.45, \bar{b}=0.5, \underline{a}=\underline{b}=0.4, A=0.16, B=0.2 .
$$

Then

$$
C=\min \{1-\bar{a}, \underline{a}, 1-\bar{b}, \underline{b}\}=0.4, K=\max \{(\bar{b}+A),(\bar{a}+B)\}<0.24 .
$$

It is very easy to verify that $\left(\mathrm{H}_{2}\right)$ holds, according to Theorem 4 , system (7) has a unique equilibrium point $z^{*}=\left\{x_{1}^{*}, x_{2}^{*}, y_{1}^{*}, y_{2}^{*}\right\}$, which is uniformly stable.

\section{Conclusion}

As is widely known, the leakage delay has a great impact on the dynamical behavior of neural networks. Thus, it is necessary and rewarding to study the leakage delay effects to the dynamic behaviors of neural networks. In this paper, we have derived some sufficient conditions ensuring the existence, uniqueness, and uniform stability of equilibrium point for fractional-order fuzzy BAM neural networks with delays in the leakage terms. We have also given an example to illustrate the feasibility and effectiveness of the obtained results. In addition, when the fractional-order differential system is equivalent to an integral one, then it is possible to extend the method to many other fractional-order fuzzy neural networks within commensurate order and fractional neutral-type fuzzy neural networks with time-varying delay in the leakage terms, which can be a good topic for further investigation.

\section{Acknowledgements}

This work is supported by National Natural Science Foundation of China (11772291), Innovation Scientists and Technicians Troop Construction Projects of Henan Province (2017JR0013). The authors would like to thank the associate editor and the referees for their detailed comments and valuable suggestions which considerably improved the presentation of the paper.

\section{References}

[1] Podlubny, I. (1999) Fractional Differential Equations. Academic Press, San Diego.

[2] Kilbas, A.A. (2006) Theory and Application of Fractional Differential Equations. Elsevier, Amsterdam.

[3] Samko, S.G., Kilbas, A.A. and Marichev, Q.I. (1993) Fractional Integrals and Derivatives. Gordon and Breach Science, Yverdon.

[4] Magin, R.L. (2006) Fractional Calculus in Bioengineering. Begell House, Connecticut.

[5] Bastos, N.R.O., Ferreira, R.A.C. and Torres, D.F.M. (2011) Discrete-Time Fractional Variational Problems. Signal Process, 91, 513-524. https://doi.org/10.1016/j.sigpro.2010.05.001

[6] Kaslika, E. and Sivasundaram, S. (2012) Nonlinear Dynamics and Chaos in Fractional Order Neural Networks. Neural Networks, 32, 245-256. https://doi.org/10.1016/j.neunet.2012.02.030

[7] Yang, X.J., Song, Q.K., Liu, Y.R. and Zhao, Z.J. (2015) Finite-Time Stability Analysis 
of Fractional-Order Neural Networks with Delay. Neurocomputing, 152, 19-26. https://doi.org/10.1016/j.neucom.2014.11.023

[8] Zhang, S., Yu, Y.G. and Wang, Q. (2016) Stability Analysis of Fractional-Order Hopfield Neural Networks with Discontinuous Activation Functions. Neurocomputing, 171, 1075-1084. https://doi.org/10.1016/j.neucom.2015.07.077

[9] Chen, L.P., Chai, Y., Wu, R.C., Ma, T.D. and Zhai, H.Z. (2013) Dynamic Analysis of a Class of Fractional-Order Neural Networks with Delay. Neurocomputing, 111, 190-194. https://doi.org/10.1016/j.neucom.2012.11.034

[10] Wang, L.M., Song, Q.K., Liu, Y.R., Zhao, Z.J. and Alsaadi, F.E. (2017) Global Asymptotic Stability of Impulsive Fractional-Order Complex-Valued Neural Networks with Time Delay. Neurocomputing, 243, 49-59. https://doi.org/10.1016/j.neucom.2017.02.086

[11] Arena, P., Fortuna, L. and Porto, D. (2000) Chaotic Behavior in Noninteger-Order Cellular Neural Networks. Physical Review E, 61, 776-781. https://doi.org/10.1103/PhysRevE.61.776

[12] Wang, Y., Zheng, C.D. and Feng, E. (2013) Stability Analysis of Mixed Recurrent Neural Networks with Time Delay in the Leakage Term under Impulsive Perturbations. Neurocomputing, 119, 454-461. https://doi.org/10.1016/j.neucom.2013.03.012

[13] Li, R.X. and Cao, J.D. (2016) Stability Analysis of Reaction-Diffusion Uncertain Memristive Neural Networks with Time-Varying Delays and Leakage Term. Applied Mathematics and Computation, 278, 54-69. https://doi.org/10.1016/j.amc.2016.01.016

[14] Kalpanaa, M. and Balasubramaniam, P. (2016) Asymptotical State Estimation of Fuzzy Cellular Neural Networks with Time Delay in the Leakage Term and Mixed Delays: Sample-Data Approach. Journal of the Egyptian Mathematical Society, 24, 143-150. https://doi.org/10.1016/j.joems.2014.07.003

[15] Liu, J. and Xu, R. (2017) Delay-Dependent Passivity and Stability Analysis for a Class of Memristor-Based Neural Networks with Time Delay in the Leakage Term. Neural Processing Letters, 46, 467-485. https://doi.org/10.1007/s11063-017-9594-6

[16] Wang, L.M., Song, Q.K., Liu, Y.R., Zhao, Z.J. and Alsaadi, F.E. (2017) Finite-Time Stability Analysis of Fractional-Order Complex-Valued Memristor-Based Neural Networks with Both Leakage and Time-Varying Delays. Neurocomputing, 245, 86-101. https://doi.org/10.1016/j.neucom.2017.03.042

[17] Yang, T., Yang, L.B., Wu, C.W. and Chua, L.O. (1996) Fuzzy Cellular Neural Networks: Theory. 1996 Fourth IEEE International Workshop on Cellular Neural Networks and Their Applications, Seville, 24-26 June 1996, 181-186.

[18] Yang, T., Yang, L.B., Wu, C.W. and Chua, L.O. (1996) Fuzzy Cellular Neural Networks: Applications. 1996 Fourth IEEE International Workshop on Cellular Neural Networks and Their Applications, Seville, 24-26 June 1996, 225-230.

[19] Ali, M.S., Balasubramaniam, P. and Zhu, Q.X. (2017) Stability of Stochastic Fuzzy BAM Neural Networks with Discrete and Distributed Time-Varying Delays. International Journal of Machine Learning and Cybernetics, 8, 263-273. https://doi.org/10.1007/s13042-014-0320-7

[20] Li, Y.K. and Li, Y.Q. (2014) Exponential Stability of BAM Fuzzy Cellular Neural Networks with Time-Varying Delays in Leakage Terms and Impulses. Abstract and Applied Analysis, 2014, 1-12.

[21] He, W.N. and Chu, L.X. (2017) Exponential Stability Criteria for Fuzzy Bidirectional Associative Memory Cohen-Grossberg Neural Networks with Mixed Delays and 
Impulses. Advances in Difference Equations, 2017, 61. https://doi.org/10.1186/s13662-017-1082-9

[22] Bao, H.M. (2016) Existence and Exponential Stability of Periodic Solution for BAM Fuzzy Cohen-Grossberg Neural Networks with Mixed Delays. Neural Processing Letters, 43, 871-885. https://doi.org/10.1007/s11063-015-9455-0

[23] Delavari, H., Baleanu, D. and Sadati, J. (2012) Stability Analysis of Caputo Fractional Order Nonlinear Systems Revisited. Nonlinear Dynamics, 67, 2433-2439. https://doi.org/10.1007/s11071-011-0157-5

[24] Li, Y., Chen, Y. and Podlubny, I. (2010) Stability of Fractional-Order Nonlinear Dynamic Systems: Lyapunov Direct Method and Generalized Mittag-Leffler Stability. Computers and Mathematics with Applications, 59, 1810-1821. https://doi.org/10.1016/j.camwa.2009.08.019

[25] Yang, T. and Yang, L. (1996) The Global Stability of Fuzzy Cellular Neural Networks. IEEE Transactions on Circuits and Systems I: Fundamental Theory and Applications, 43, 880-883. https://doi.org/10.1109/81.538999 